\title{
Avaliação do teor de sódio em salgadinhos comerciais e da rotulagem de acordo com a RDC $n^{\circ}$ 26/2015 sobre alergênicos alimentares
}

\section{Assessment of sodium content and labelling of commercial snack in accordance with RDC 26/2015 on allergens}

\author{
Valriane Suelen Reis ${ }^{1}$, Nelson Consolin Filho², Michel Rocha Baqueta ${ }^{3 *}$ (i), \\ Bogdan Demczuk Junior ${ }^{1}$ \\ 1Universidade Tecnológica Federal do Paraná (UTFPR), Departamento Acadêmico de Alimentos (DALIM), Campus \\ Campo Mourão, Campo Mourão/PR - Brasil \\ ${ }^{2}$ Universidade Tecnológica Federal do Paraná (UTFPR), Coordenação do Curso de Licenciatura em Química \\ (COLIQ), Campus Campo Mourão, Campo Mourão/PR - Brasil \\ ${ }^{3}$ Universidade Tecnológica Federal do Paraná (UTFPR), Programa de Pós-graduação em Tecnologia de Alimentos \\ (PPGTA), Campus Campo Mourão, Campo Mourão/PR - Brasil
}

*Corresponding Author: Michel Rocha Baqueta, Universidade Tecnológica Federal do Paraná (UTFPR),

Programa de Pós-Graduação em Tecnologia de Alimentos (PPGTA), Campus Campo Mourão, via Rosalina Maria dos Santos, 1233, Caixa Postal: 271, CEP: 87301-899, Campo Mourão/PR - Brasil, e-mail:

michelbaqueta@gmail.com

Cite as: Reis, V. S., Consolin Filho, N., Baqueta, M. R., \& Demczuk Junior, B. (2020). Assessment of sodium content and labelling of commercial snack in accordance with RDC 26/2015 on allergens. Brazilian Journal of Food Technology, 23, e2019093. https://doi.org/10.1590/1981-6723.09319

\begin{abstract}
Resumo
Os alimentos extrusados snacks são consumidos mundialmente por causa da variedade de formas, texturas e sabores, além da praticidade no consumo. Contudo, a maioria das formulações possui valor biológico reduzido e elevados teores de cloreto de sódio $(\mathrm{NaCl})$. Atualmente, no Brasil, além das informações nutricionais, é obrigatório informar ao consumidor a presença de alimentos alergênicos. Alguns requisitos sobre esse assunto foram estabelecidos pela RDC $n^{\circ} 26$, de 2 de julho de 2015. Desde a regularização dessa resolução, existe uma falta de estudos com foco na avaliação da rotulagem de diversos alimentos, incluindo os snacks. Assim, o presente estudo teve dois objetivos: verificar os rótulos de snacks de diferentes marcas comerciais de acordo com a RDC n 26/2015 sobre alergênicos e determinar o teor de sódio, comparando-o com a descrição na informação nutricional fornecida na embalagem dos produtos. A avaliação da rotulagem dos "snacks" extrusados encontrou inconformidades em 4 marcas das 16 analisadas. Com base na RDC n 26/2015 sobre alergênicos alimentares, foram constatadas ausências de informações obrigatórias relacionadas a ingredientes alergênicos, como a farinha de trigo. Foi verificado que $87,5 \%$ dos snacks continham algum alergênico e que apenas $68,75 \%$ dos rótulos estavam de acordo com a RDC n 26/2015. Além disso, foi identificado que, na maioria dos rótulos, falta a informação sobre contaminação cruzada por alergênicos no processamento dos produtos. Quanto à análise de sódio por espectrometria de absorção atômica com chama, os resultados obtidos mostraram que, na maioria das amostras, a concentração de sódio era elevada e que diferia dos valores indicados na tabela nutricional das embalagens.
\end{abstract}


Portanto, a adição elevada de sódio na forma de cloreto de sódio nos "snacks" extrusados pode contribuir para exceder a ingestão diária recomendada e, consequentemente, aumentar a disponibilidade para decorrência de riscos de doenças cardiovasculares, coronárias, entre outras, associadas ao consumo de $\mathrm{NaCl}$ em excesso.

Palavras-chave: Extrusado; Saúde; Alergia; Legislação; Embalagem; $\mathrm{NaCl}$.

\begin{abstract}
Extruded snack foods are consumed worldwide due to the variety of shapes, textures and flavors, in addition to the convenience in consumption. However, most formulations have a reduced biological value and high levels of sodium chloride $(\mathrm{NaCl})$. Currently, in Brazil, in addition to nutritional information, it is necessary to inform the consumer about the presence of allergenic foods. Some requirements on this subject were established by RDC No. 26 of July from 2015. Since the regularization of RDC No. 26/2015, there has been a lack of studies focusing on the evaluation of the labeling of various foods, including snacks. Thus, the present study had two objectives: to verify the labels of snacks of different commercial brands according to RDC No. 26 of 2015 on allergens, and to determine the sodium content and compare it with the description in the nutritional information provided on the packaging of products. The evaluation of the labelling of extruded snacks found non-conformities in four brands of the sixteen analyzed. Based on RDC No. 26 of 2015 on food allergens, it was found that there was a lack of mandatory information related to allergenic ingredients, such as wheat flour. It was found that $87.5 \%$ of snacks contained some allergens and that only $68.75 \%$ of the labels were in accordance with RDC No. 26/2015. In addition, it was identified that most of the labels lacked information on cross-contamination by allergens in the processing of the products. Regarding the sodium analysis, the results obtained by flame atomic absorption spectrometry showed that in most samples, the sodium concentration was high and that it differed from the values indicated in the nutritional table of the packages. Therefore, the high addition of sodium in the form of sodium chloride in the extruded snacks may contribute to exceeding the recommended daily intake and, consequently, increase the availability for risks of cardiovascular and coronary diseases, among others, associated with the excessive consumption of $\mathrm{NaCl}$.
\end{abstract}

Keywords: Extruded; Health; Allergy; Legislation; Packaging; $\mathrm{NaCl}$.

\title{
1 Introdução
}

Os alimentos extrusados snacks são consumidos mundialmente por causa da variedade de formas, texturas e sabores, além da praticidade no consumo. A maioria das formulações possui valor biológico reduzido, com baixa concentração de nutrientes, além de ser fabricada a partir de cereais transgênicos e conter elevados teores de sódio (Lucas et al., 2016).

Diversos materiais são utilizados na elaboração de produtos extrusados expandidos. Isso inclui grits de milho e farinhas de arroz, milho, trigo, soja, fécula e farinha de mandioca (Trombini et al., 2013). O uso de outros materiais, como bagaço de malte (Bieli et al., 2015), casca de soja (Carvalho et al., 2005), fragmentos de feijão (Soares Júnior et al., 2011) e farinha de cará (Alves \& Grossmann; 2002) também são comuns. Contudo, o milho e o trigo são os principais materiais na produção de expandidos por extrusão, tanto para snacks como para farinhas instantâneas (Carvalho et al., 2005).

A comercialização de snacks, também chamados de "salgadinhos", apresenta um faturamento anual de aproximadamente R\$ 1 bilhão, com produção em torno de 80 mil toneladas ao ano no Brasil. No mercado mundial, as estimativas mostram um progresso anual (Poltronieri et al., 2000; Capriles \& Arêas, 2012). No mesmo sentido, existe um acréscimo na instalação de linhas de produção de snacks com apelos nutricionais, fortificados, com baixo teor de gordura e/ou funcionais (Trombini et al., 2013). Um exemplo disso é a produção de snacks extrusados com adição de spirulina para melhorar a qualidade nutricional das formulações (Joshi et al., 2012). 
A tecnologia de extrusão de alimentos tem sido amplamente utilizada para produzir cereais prontos e alimentos para "salgadinhos" (Trombini et al., 2013; Bieli et al., 2015). As características físicas (expansão, dureza e densidade) de um produto extrusado, como os snacks, são parâmetros importantes em termos de aceitabilidade do consumidor, bem como propriedades funcionais do produto final (Mendonça et al., 2000). Os salgadinhos extrusados mais comuns são predominantemente feitos de farinha de cereais (trigo e/ou milho) em razão de suas boas características de expansão. No entanto, produtos alimentícios extrusados produzidos a partir de cereais tendem a ter baixo teor de proteína e um baixo valor biológico por causa de seu conteúdo limitado de aminoácidos essenciais (Joshi et al., 2012). Após a extrusão das farinhas amiláceas, o processo segue pela secagem e adição de aromatizantes (Mitchell \& Arêas, 1992; Capriles \& Arêas, 2005).

A farinha de trigo, em particular, é a principal fonte de glúten em um alimento processado, como os snacks. Além dela, por causa do cultivo de outras culturas na mesma plantação, por exemplo, milho, soja e arroz, pode ocorrer uma contaminação entre os grãos de diferentes culturas, levando à presença de traços de trigo. Outro problema associado à contaminação cruzada com glúten é na linha de produção industrial. Um mesmo equipamento pode ser utilizado para o processamento de múltiplos alimentos. Por isso, a presença de glúten em alimentos deve ser informada em alimentos e bebidas, uma vez que, como uma reação alérgica severa, a alergia ao glúten pode levar a diversas manifestações no indivíduo, causando até mesmo a morte.

A alergia alimentar é uma reação adversa a um dado alimento e ocorre a partir de uma resposta imune específica do sistema imunológico de indivíduos alérgicos (Muraro et al., 2014). Entre as reações que a alergia alimentar pode causar, a mais preocupante é a anafilaxia, uma reação aguda que afeta dois ou mais sistemas de órgãos e que pode ser fatal (Simons \& Sampson, 2015). De acordo com a Agência Nacional de Vigilância Sanitária e a Comissão do Codex Alimentarius, cerca de 160 alimentos podem ser causadores de alergia alimentar (Codex Alimentarius Commission, 2008; Brasil, 2015).

A maneira mais efetiva de o consumidor obter informações sobre o produto que pretende comprar é a embalagem. O rótulo de um alimento embalado permite uma comunicação com o consumidor a respeito de diversas características relacionadas ao produto, como informações sobre ingredientes, nutrientes e quantidades (Smith, 2010). Atualmente, no Brasil, é obrigatório declarar ao consumidor a presença de alimentos alergênicos (Brasil, 2002, 2003; Pieretti et al., 2009). Alguns requisitos para a rotulagem obrigatória de alimentos industrializados com alergênicos alimentares foram estabelecidos pela $R D C \mathrm{n}^{\circ} 26$, de 2 de julho de 2015, uma normativa recente e de valor social agregado, principalmente ao grupo de pessoas com alergia alimentar (Brasil, 2015). Essa resolução dispõe sobre os requisitos para rotulagem obrigatória dos principais alimentos que causam alergias. $O$ prazo para a adequação das indústrias de alimentos às novas normas de rotulagem estabelecidas pela Agência Nacional de Vigilância Sanitária (ANVISA) encerrou-se em julho de 2016.

Apesar de escassos, estudos evidenciando o descumprimento dos parâmetros estabelecidos pela legislação no que diz respeito à rotulagem de alimentos com ingredientes potencialmente alergênicos têm sido desenvolvidos, mesmo após o fim do prazo de adequação. Por exemplo, Farias et al. (2017) avaliaram os rótulos de alimentos derivados de leite e constataram que $92 \%$ dos casos apresentaram não conformidades com a RDC n 26/2015. Outro exemplo é o estudo de Zorzanello \& Weschenfelder (2017), que, após avaliação da rotulagem de pães integrais, verificaram que $16,7 \%$ dos rótulos não mencionavam presença dos ingredientes alergênicos. Mais recentemente, Santana (2018) verificou que 11\% dos rótulos de chocolates analisados não apresentavam a declaração para alergênico, mesmo havendo ingredientes com obrigatoriedade de declaração. Pelo menos uma não conformidade com a RDC n 26/2015 foi encontrada em 53\% dos rótulos de chocolate, cujas categorias "chocolate diet/light/zero" e "confeitos" apresentaram não conformidade em $100 \%$ e $50 \%$ dos rótulos, respectivamente. 
Além dos alergênicos, as embalagens dos alimentos devem conter outras informações nutricionais, como fibras, minerais, gorduras etc. Um dos micronutrientes que deve ser informado é a quantidade de sódio. $\mathrm{O}$ sódio em alimentos, adicionado na forma de cloreto de sódio $(\mathrm{NaCl})$ e popularmente conhecido como sal ou sal de cozinha, é um dos minerais preocupantes na dieta humana, apesar de, em sua forma elementar, ser essencial para o desenvolvimento e funcionamento de algumas vias metabólicas. Além da função de dar sabor e realçar o sabor salgado, o cloreto de sódio é usado desde a Antiguidade como conservante (Beauchamp \& Mennella, 2009). Contudo, o seu consumo em excesso é associado a diversos problemas de saúde, incluindo hipertensão, doenças cardiovasculares, câncer gástrico, deficiência nos rins e aumento da gravidade em casos de asma (He \& MacGregor, 2009; Doyle \& Glass, 2010).

O teor de cloreto de sódio em alimentos e as alergias alimentares são considerados questões de saúde pública mundial. Alimentos extrusados snacks, como os salgadinhos comerciais, além de serem conhecidos por seu alto teor de cloreto de sódio, são fabricados na maioria das vezes com farinhas amiláceas, por exemplo, o trigo. Portanto, um estudo abordando a avaliação do teor de sódio e a adequação de acordo a legislação atual sobre alergênicos ( $\mathrm{RDC} \mathrm{n}^{\circ}$ 26/2015), em conjunto com uma análise de dados apropriada, parece ser uma abordagem adequada para fins de segurança e saúde alimentar.

Desde a regularização da $\mathrm{RDC} \mathrm{n}^{\circ}$ 26/2015 sobre alergênicos, existe um número escasso de pesquisas com foco na avaliação da rotulagem de diversos alimentos, incluindo os snacks. Assim, o presente estudo teve o objetivo de verificar rótulos de snacks de diferentes marcas comerciais de acordo com a RDC no 26/2015 sobre alergênicos, além de determinar o teor de sódio experimentalmente e compará-lo com a descrição na informação nutricional fornecida na embalagem dos produtos.

\section{Material e métodos}

\subsection{Amostras}

As amostras de salgadinhos extrusados foram obtidas de um único lote no comércio local das cidades de Campo Mourão $(n=5)$, Irati $(n=2)$ e Curitiba $(n=9)$, no estado do Paraná, Brasil. Do total de amostras, 8 eram à base de trigo, 6 , de milho, 1 , de batata, e 1, de uma mistura de cereais (trigo, milho, aveia e arroz integral). Na escolha das marcas analisadas, não houve distinção entre produtos regionais ou de marcas de reconhecimento nacional.

\subsection{Análise da adequação à RDC nº 26/2015}

Foi desenvolvido um instrumento de coleta de dados (Quadro 1) considerando as regulamentações para os ingredientes alergênicos constantes na RDC $n^{\circ}$ 26/2015. Cada embalagem foi codificada com números sequenciais ( 1 a 16) de acordo com a marca e analisada individualmente. As questões referentes à adequação dos rótulos foram assinaladas com as respostas "SIM", "NÃO" e "NÃO SE APLICA". Além disso, o contraste entre a embalagem e o conteúdo impresso, um requisito também estabelecido pela legislação, foi verificado. Nessa etapa, também foram coletadas informações da tabela nutricional sobre o teor de sódio por porção. 
Reis, V. S. et al.

Quadro 1. Instrumento de coleta de dados das informações da embalagem.

\begin{tabular}{|c|c|c|c|}
\hline \multicolumn{4}{|l|}{$\begin{array}{l}\text { Codificação: } \\
\text { Nome comercial: } \\
\text { Peso líquido: } \\
\text { Data de fabricação: } \\
\text { Data de vencimento: } \\
\text { Descrição do produto: } \\
\text { Local de fabricação: } \\
\text { Teor de sódio (mg): } \\
\text { Outras informações importantes: }\end{array}$} \\
\hline ITENS DE VERIFICAÇÃO & SIM & NÃO & NA \\
\hline $\begin{array}{l}\text { 1) Entre os ingredientes informados na embalagem, há pelo menos um dos } \\
\text { informados no anexo da RDC? }\end{array}$ & & & \\
\hline $\begin{array}{l}\text { 2) O rótulo do produto, que contém ingredientes listados no anexo da RDC, traz } \\
\text { as seguintes declarações: "Alérgicos: contém" ou "Alérgicos: pode conter"? }\end{array}$ & & & \\
\hline 3) O rótulo informa sobre a contaminação cruzada no processamento do produto? & & & \\
\hline $\begin{array}{l}\text { 4) As advertências exigidas nos artigos } 6^{\circ} \text { e } 7^{\circ} \text { da RDC estão agrupadas } \\
\text { imediatamente depois ou abaixo da lista de ingredientes e com caracteres legíveis? }\end{array}$ & & & \\
\hline a) Caixa-alta & & & \\
\hline b) Negrito & & & \\
\hline c) Cor contrastante com o fundo do rótulo & & & \\
\hline $\begin{array}{l}\text { d) Altura mínima de } 2 \mathrm{~mm} \text { e não inferior à altura de letra utilizada na lista de } \\
\text { ingredientes }\end{array}$ & & & \\
\hline 5) As declarações exigidas estão dispostas em locais encobertos? & & & \\
\hline $\begin{array}{l}\text { 6) No caso das embalagens com área de painel principal igual ou inferior a } \\
0,01 \mathrm{~m}^{2} \text {, a altura mínima dos caracteres é de } 1 \mathrm{~mm} \text { ? }\end{array}$ & & & \\
\hline $\begin{array}{l}\text { 7) Os alimentos, ingredientes, aditivos alimentares e coadjuvantes de tecnologia } \\
\text { veiculam algum tipo de alegação relacionada à ausência de alimentos alergênicos } \\
\text { ou alérgenos alimentares? }\end{array}$ & & & \\
\hline
\end{tabular}

\subsection{Determinação do teor de sódio}

A concentração de sódio foi realizada utilizando a técnica de espectrometria de absorção atômica com chama (FAAS), utilizando um espectrofotômetro da marca Analytik Jena®, modelo novAA 300. A metodologia de preparo de amostra foi descrita por Jorhem et al. (2000) e aplicada para uma amostra de cada snack representativa de todo o conteúdo da embalagem. Foram pesadas aproximadamente $3 \mathrm{~g}$ de amostra de salgadinho extrusado em cadinhos previamente secos. As amostras foram calcinadas em forno mufla marca Coel a $500{ }^{\circ} \mathrm{C}$ por um período de 4 horas. As cinzas resultantes foram submetidas à digestão ácida com $5 \mathrm{~mL}$ de ácido clorídrico concentrado e evaporadas em um bloco de aquecimento. Após esse procedimento, os cadinhos foram lavados com $25 \mathrm{~mL}$ de ácido nítrico PA com concentração de $65 \%$. As soluções foram preparadas a partir da diluição de $0,5 \mathrm{~mL}$ do ácido digerido em balões volumétricos de $100 \mathrm{~mL}$ contendo água destilada para obter-se uma diluição de 1:200. Para a calibração do equipamento de absorção atômica, foi preparada uma curva de solução padrão com concentração de sódio PA na faixa de $10 \mathrm{mg} / \mathrm{L}$ a $0,1 \mathrm{mg} / \mathrm{L}$. Os resultados de teor de sódio foram expressos em média da duplicata obtida. 


\subsection{Análise estatística}

Todas as informações relativas às adequações dos rótulos registradas nos instrumentos de coleta de dados e determinação do teor de sódio foram analisadas comparativamente por meio de gráficos e tabelas, utilizando o Microsoft Excel, versão 2013.

\section{Resultados e discussão}

Entre os 16 rótulos de snacks extrusados analisados foi verificado que 3 deles ainda não estavam de acordo as disposições da $\mathrm{RDC} \mathrm{n}^{\circ}$ 26/2015 sobre alergênicos alimentares. Foi constatado que na amostra 8, fabricada com farinha de trigo, não havia a informação "contém glúten". Nessa situação, fica evidente o risco exposto ao consumidor com alergia ao glúten. Em geral, esses rótulos não apresentavam a declaração para alergênicos ou nenhuma indicação de alerta, embora houvesse a presença de ingredientes com obrigatoriedade de declaração. Em amostras de chocolates nacionais e importados, Santana (2018) encontrou irregularidades em $11 \%$ dos rótulos analisados. Contudo, um resultado interessante é que $19 \%$ dos chocolates importados apresentavam irregularidades conforme a RDC $n^{\circ}$ 26/2015 contra 9\% nacionais. Apesar de haver ainda irregularidades quanto à declaração de alergênicos, este estudo mostra que o Brasil está se atentando aos parâmetros da legislação. $\mathrm{O}$ autor também aponta que alimentos importados estão mais susceptíveis a reações de alergia alimentar por falta de advertência no rótulo. A ausência de declaração para alergênicos também foi destacada em um estudo desenvolvido por Farias et al. (2017), que avaliaram rótulos de produtos lácteos. No mesmo sentido, Zorzanello \& Weschenfelder (2017) verificaram que 16,7\% dos rótulos de pães integrais não apresentavam a declaração para alergênicos.

No caso da amostra 5, foi verificado que, além da falta de declaração sobre a presença da declaração "contém glúten" ou "pode conter glúten", como no caso anterior, a cor das letras não estava em contraste com o fundo da embalagem, dificultando a visualização dos ingredientes pelo consumidor. Hefle et al. (2007) notaram que a expressão "pode conter alergênicos" no rótulo de alimentos é uma expressão às vezes ignorada por indivíduos com distúrbios de alergia alimentar. A justificativa é que esses consumidores acreditam, muitas vezes, que não existem riscos ao consumir esses produtos. Todavia, em alimentos analisados com o rótulo "pode conter traços de amendoim" por Hefle et al. (2007), os autores encontraram níveis detectáveis de amendoim, e, por essa razão, foi comprovado o risco aos consumidores alérgicos. Além disso, o acesso à informação é importante para garantir a saúde do consumidor. Assim, é essencial que o alerta para alérgenos esteja em local de fácil visualização, não encoberto e distante do local de selagem, conforme estabelece a legislação (Santana, 2018). Neste contexto, um estudo desenvolvido com idosos mostrou que $82 \%$ deles consideravam as informações contidas nos rótulos dos alimentos de difícil leitura e compreensão (Nascimento et al., 2014). Apesar de os snacks serem consumidos em grande parte por crianças, estudos indicaram que a alergia alimentar é mais prevalente nelas do que em adultos (Berzuino et al., 2017), porém o alerta de alimentos alérgenos se estende a todos os indivíduos, uma vez que as consequências podem ser fatais.

Na análise do rótulo da amostra 6, foi verificada a seguinte alegação: "Alérgicos: não contém alergênicos". De acordo com o artigo 9 da RDC n⿳ 26/2015 sobre alergênicos alimentares, não pode ser veiculado qualquer tipo de alegação relacionada à ausência de alergênicos. Somente pode ser declarada a presença, quando houver. Na amostra 13, outras informações relacionadas à rotulagem foram encontradas inadequadas: a unidade de medida do teor de sódio estava errada e a informação de peso da embalagem e a informação de porção eram desconformes. Neste último caso, o painel frontal da embalagem trazia a informação de peso líquido de $70 \mathrm{~g}$, e na quantidade de sódio na tabela nutricional eram informados $186 \mathrm{~g}$ por porção de $25 \mathrm{~g}$. De acordo com a RDC n $\mathrm{n}^{\circ} 360 / 2003$, as unidades nutricionais que devem constar na rotulagem, no caso do sódio, devem ser expressas em miligramas, e não em gramas, como foi verificado na embalagem. 
Nos últimos anos, um número restrito de estudos tem sido desenvolvido abordando a rotulagem de alimentos alergênicos (Pieretti et al., 2009). Pelo fato de a alergia alimentar ser reconhecida como um problema de saúde pública mundial, inevitavelmente existem diferentes abordagens para rotulagens de alimentos no mundo. No Brasil, embora seja recente em termos de institucionalização, a RDC no 26/2015 traz um assunto atual e recorrente. A alergia alimentar causa severas complicações que podem levar até a morte. De tal forma, a adequação da informação sobre ingredientes alérgenos em alimentos, principalmente processados, é questão essencial para o público com alergia alimentar. Portanto, cuidar das informações relativas ao rótulo dos alimentos em todo setor, particularmente dos salgadinhos snacks, é uma demanda mundial necessária, e, no Brasil, este estudo reporta essas falhas existentes mesmo alguns anos após publicação da RDC sobre alergênicos em alimentos.

A quantidade de irregularidades encontradas nas rotulagens das amostras de snacks foi reunida na Figura 1, por meio das porcentagens de adequação para os parâmetros da legislação. Foi verificado que, entre os ingredientes informados na embalagem, $87,5 \%$ das amostras havia pelo menos um alergênico declarado entre os ingredientes. Mesmo contendo pelo menos um dos ingredientes listados no anexo da RDC n $n^{\circ}$ 26/2015, pouco mais da metade $(68,75 \%)$ dos rótulos trazia as declarações "Alérgicos: contém" ou "Alérgicos: pode conter".

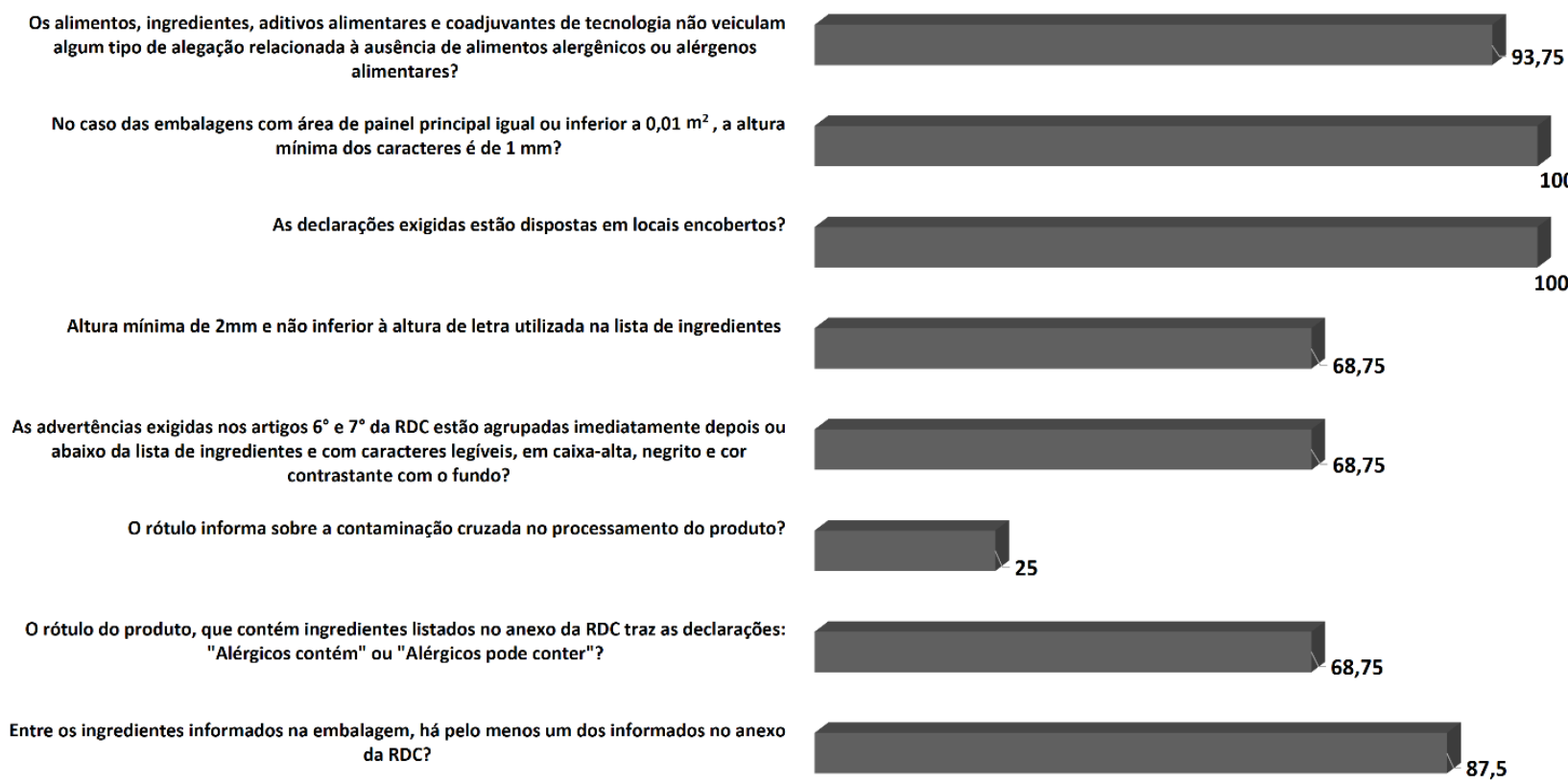

Figura 1. Porcentagem de adequação aos parâmetros da legislação sobre alergênicos nos rótulos de salgadinhos.

Com relação à informação sobre contaminação cruzada com alergênicos durante o processamento do produto, apenas $25 \%$ dos rótulos analisados trazia essa declaração. Uma maneira de advertir o consumidor nesse caso seria a expressão "pode conter trigo". Foi verificado também que as advertências exigidas na RDC $n^{\circ}$ 26/2015 atendem aos requisitos em $68,75 \%$ dos rótulos analisados. Entre os requisitos, é necessário que as advertências estejam agrupadas imediatamente depois ou abaixo da lista de ingredientes e que os caracteres estejam impressos de forma legível, em caixa-alta, destacados em negrito e em cor contrastante com a cor de fundo da embalagem. Quanto à adequação dos caracteres, $68,75 \%$ dos rótulos analisados continham letras com altura mínima de $2 \mathrm{~mm}$ e não inferior à altura da letra da lista de ingredientes.

Nenhuma das declarações exigidas estava disposta em locais encobertos, como dobras ou selagens, por exemplo. Todas as embalagens analisadas apresentavam caracteres com altura mínima de $1 \mathrm{~mm}$ no caso do painel principal (área igual ou inferior a $0,01 \mathrm{~m}^{2}$ ), em conformidade com a legislação. Das 16 amostras 
analisadas, apenas $1(6,25 \%)$ trazia a alegação vinculada à ausência de alergênicos, uma informação desnecessária perante as especificações da legislação. As demais (93,75\%) estavam coerentes e sem informações redundantes.

Na literatura, Gendel (2012) investigou as diferentes rotulagens de alimentos alergênicos e descobriu que as estruturas de rotulagem relacionada a ingredientes alergênicos diferem significativamente ao redor do mundo. Em cada país, uma jurisdição identifica uma prioridade diferente de alérgenos, e, muitas vezes, não são totalmente esclarecidos os critérios utilizados para desenvolver as listas de alérgenos prioritários. Além disso, os padrões para adicionar e remover ingredientes dos regulamentos diferem também. Lista de prioridade de alergênico, processos de adequação de rótulos e lista de classificação contendo os alérgenos de maior preocupação foram as informações menos mencionadas na maioria dos rótulos.

Entre os demais ingredientes utilizados na fabricação de salgadinhos extrusados pode ser citado o cloreto de sódio. O sódio é um mineral essencial para o funcionamento adequado do corpo humano, contudo, na forma de sal $(\mathrm{NaCl})$, a ingestão em excesso oferece inúmeros riscos à saúde. O consumo excessivo de cloreto de sódio tem sido associado principalmente à ocorrência de doenças cardiovasculares, como aumento da pressão arterial. Além disso, $62 \%$ dos acidentes vasculares cerebrais e $49 \%$ das doenças coronárias são relacionados ao consumo de cloreto de sódio em excesso, bem como a outros efeitos negativos à saúde, incluindo câncer gástrico, diminuição da densidade mineral óssea e obesidade (Tsugane et al., 2004; He \& MacGregor, 2010).

No metabolismo, o mineral sódio participa regulando o volume extracelular, mantém o equilíbrio ácidobase, a transmissão neural, a função renal, o débito cardíaco e a contração miocítica. A Organização Mundial da Saúde indica que, para prevenir doenças crônicas, a ingestão diária máxima de sódio por adultos deve ser inferior a $5 \mathrm{~g}$ por dia. Contradizendo essas recomendações, a ingestão média de sódio nos Estados Unidos é estimada em 8,2 até 9,4 g por dia, no Reino Unido, em 9,4 g, e nos países asiáticos, em mais de $12 \mathrm{~g}$ (Liem et al., 2011). No Brasil, o valor recomendado também é excedido, chegando até $16,7 \mathrm{~g}$ de sódio consumido por pessoa em um dia (Feitosa \& Silva, 2014). Nos países ocidentais, aproximadamente $75 \%$ do sódio na dieta vem de alimentos processados e consumidos fora de casa, como os salgadinhos snacks, já que o processamento envolve a adição de sódio por razões de sabor e conservação (Liem et al., 2011).

De acordo com regulamentações brasileiras (Brasil, 2010), o valor diário de referência (VDR) para o consumo de sódio é $2.400 \mathrm{mg}$. Dessa forma, a ingestão de um pacote médio de salgadinhos snacks (110 g), por exemplo, significa aproximadamente $10 \%$ do VDR para sódio $(241,8 \mathrm{mg})$. Neste estudo, os cálculos para se chegar aos valores em relação a $100 \mathrm{~g}$ foram feitos a partir das informações existentes nos rótulos e com a quantidade de sódio existente em cada porção.

Na Tabela 1 são apresentados os resultados das concentrações de sódio das 16 marcas de salgadinhos snacks medidos experimentalmente pela análise FAAS. Para fins de comparação, constam também os valores de sódio informados pelo fabricante no rótulo dos produtos. Também é possível verificar na mesma tabela o número de não conformidades com relação à rotulagem de alergênicos. A análise dos resultados mostrou que os valores obtidos pela FAAS foram superiores aos informados nos rótulos dos produtos na maioria dos casos, exceto para as amostras 10 e 16, que tiveram valores inferiores aos informados nos rótulos. A quantidade média de sódio encontrada foi de $1.785,188 \mathrm{mg} / 100 \mathrm{~g}$ de amostra, com resultados variando entre $886,667 \mathrm{mg} / 100 \mathrm{~g}$ e $2.770,000 \mathrm{mg} / 100 \mathrm{~g}$. Em contraste, os resultados de acordo com a informação nutricional indicavam que as amostras continham em média $981,025 \mathrm{mg} / 100 \mathrm{~g}$ de sódio, com resultados variando entre $596 \mathrm{mg}$ e $1.368,2 \mathrm{mg} / 100 \mathrm{~g}$ de amostra. 
Tabela 1. Comparação do teor de sódio obtido experimentalmente e fornecido na rotulagem, e número de não conformidades nos snacks.

\begin{tabular}{|c|c|c|c|c|}
\hline Amostra & $\begin{array}{c}\text { Teor de sódio } \\
\text { experimental } \\
(\mathrm{mg} / 100 \mathrm{~g})\end{array}$ & $\begin{array}{l}\text { Teor de sódio no rótulo } \\
\qquad(\mathrm{mg} / 100 \mathrm{~g})\end{array}$ & $\begin{array}{c}\text { Diferença }(\%) \text { entre } \\
\text { rotulagem e } \\
\text { experimental }\end{array}$ & $\begin{array}{l}\text { Número de não } \\
\text { conformidades }\end{array}$ \\
\hline 1 & $1533,0 \pm 1,0$ & 692,0 & 121,5 & 1 \\
\hline 2 & $1569,6 \pm 0,1$ & 748,0 & 109,8 & 0 \\
\hline 3 & $2770,0 \pm 0,2$ & 696,0 & 297,9 & 1 \\
\hline 4 & $2428,3 \pm 0,2$ & 1144,0 & 112,2 & 1 \\
\hline 5 & $2131,6 \pm 0,3$ & 1368,2 & 55,8 & 4 \\
\hline 6 & $1748,3 \pm 0,1$ & 596,0 & 193,3 & 1 \\
\hline 7 & $2663,3 \pm 0,5$ & 1072,0 & 148,4 & 0 \\
\hline 8 & $2543,3 \pm 0,1$ & 1360,0 & 87,0 & 5 \\
\hline 9 & $2670,0 \pm 0,6$ & 1536,0 & 73,8 & 0 \\
\hline 10 & $886,7 \pm 0,2$ & 1368,2 & $-35,2$ & 5 \\
\hline 11 & $1035,5 \pm 0,1$ & 692,0 & 49,6 & 0 \\
\hline 12 & $1245,0 \pm 0,2$ & 656,0 & 89,8 & 1 \\
\hline 13 & $1891,6 \pm 0,1$ & 744,0 & 154,2 & 1 \\
\hline 14 & $1166,6 \pm 0,5$ & 964,0 & 21,0 & 1 \\
\hline 15 & $1066,1 \pm 0,2$ & 744,0 & 43,3 & 1 \\
\hline 16 & $1213,6 \pm 0,4$ & 1316,0 & $-7,8$ & 0 \\
\hline Média & 1785,1 & 981,1 & - & - \\
\hline
\end{tabular}

Na Tabela 1 também são mostradas as diferenças percentuais dos resultados obtidos em relação aos valores declarados no rótulo. De acordo com a RDC n $\mathrm{n}^{\circ} 360 / 2003$, é admitida para os valores analíticos uma tolerância de $\pm 20 \%$ em relação aos valores de nutrientes declarados no rótulo (Brasil, 2003). A análise dos dados mostrou que, das 16 marcas de salgadinhos analisadas, 14 não estavam de acordo com a legislação e ultrapassavam largamente os valores de tolerância recomendados pela legislação. Em algumas amostras $(1,2,3,4,6,7$ e 13), a comparação dos valores indicou uma diferença maior que $100 \%$ no teor de sódio em relação à informação nutricional dessas amostras. Apenas as amostras 14 e 16 apresentaram concordância com as concentrações de sódio indicadas no rótulo e de acordo com a $\mathrm{RDC} \mathrm{n}^{\circ}$ 360/2003.

Feitosa \& Silva (2014) avaliaram o teor de sódio em salgadinhos extrusados utilizando como ferramenta a tabela nutricional e encontraram um teor médio de 812 a $1.018 \mathrm{mg} / 100 \mathrm{~g}$ de sódio, o que corrobora os resultados encontrados no presente estudo usando a informação nutricional. Contudo, analisando apenas a informação nutricional, não é possível afirmar que esses valores são completamente confiáveis. Estudos mostram que técnicas analíticas, como a espectrometria de absorção atômica com chama, são mais adequadas para esse fim. Além disso, pode haver uma grande diferença inter e intralote nos teores de sódio, pois a adição de cloreto de sódio pode variar consideravelmente de produto para produto (Domingues Junior, 2012; Silva, 2014).

Recentemente, Gonçalves et al. (2016) analisaram seis marcas de salgadinhos e encontraram um teor médio de 484,15 mg de sódio na porção de $100 \mathrm{~g}$ de amostra pelo método de fotometria de chama. Em outro estudo realizado pela ANVISA utilizando a técnica de espectrometria de emissão óptica com plasma de 
argônio indutivamente acoplado e a FAAS, as amostras apresentaram em média $715 \mathrm{mg}$ de sódio em $100 \mathrm{~g}$ em 14 salgadinhos snacks (Brasil, 2013).

Em geral, durante o levantamento de literatura científica sobre salgadinhos snacks realizado aqui, foi verificada uma escassez de trabalhos publicados que abordassem a análise de rotulagens de alimentos. Quanto à literatura científica sobre análise de rotulagem de alimentos processados contendo alergênicos, não foi encontrado nenhum trabalho publicado, principalmente em se tratando de salgadinhos snacks. Por essa razão, é considerada relevante a realização de mais pesquisas com esse objetivo, uma vez que a oferta e a procura por alimentos com especificações adequadas sobre alergia alimentar e com teores de sódio reduzido são cada vez mais uma demanda mundial.

\section{Conclusão}

A avaliação da rotulagem dos "snacks" extrusados encontrou inconformidades em 4 marcas das 16 analisadas. Com base na RDC n ${ }^{\circ}$ 26/2015 sobre alergênicos alimentares, foram constatadas ausências de informações obrigatórias relacionadas a ingredientes alergênicos, como a farinha de trigo. Além disso, foi identificada, na maioria das amostras analisadas, falta de informação sobre contaminação cruzada por alergênicos no processamento dos produtos.

Quanto à análise de sódio, os resultados obtidos experimentalmente mostraram que, na maioria dos "snacks" extrusados, a concentração de sódio diferia em grandes proporções dos valores indicados na tabela nutricional das embalagens. Apesar de esses resultados serem significativos, novos estudos com um número maior de amostras e replicatas devem ser realizados para confirmar esses resultados. Contudo, é conhecido que a ingestão elevada de sódio na forma de cloreto de sódio em alimentos industrializados, como os "snacks" extrusados, pode contribuir para exceder a ingestão diária recomendada e, consequentemente, aumentar a disponibilidade para ocorrência de doenças cardiovasculares, coronárias, entre outras, associadas ao consumo em excesso de $\mathrm{NaCl}$.

\section{Referências}

Alves, R. M. L., \& Grossmann, M. V. E. (2002). Parâmetros de estrusão para produção de "snacks" de farinha de cará (Dioscorea alata). Food Science and Technology (Campinas), 22(1), http://dx.doi.org/10.1590/S0101-20612002000100006 Beauchamp, G. K., \& Mennella, J. A. (2009). Early flavor learning and its impact on later feeding behavior. Journal of Pediatric Gastroenterology and Nutrition, 48(Supl.1), 25-30. PMid:19214055. http://dx.doi.org/10.1097/MPG.0b013e31819774a5

Berzuino, M. B., Fernandes, R. C. S., Lima, M. A., Matias, A. C. S., \& Pereira, I. R. O. (2017). Alergia alimentar e o cenário regulatório no Brasil. Revista Eletrônica de Farmácia, 14(2), 23-36. http://dx.doi.org/10.5216/ref.v14i2.43433

Bieli, B. C., Marques, D. R., Marchi, L. B., Quelhas, J. O. F., Chinellato, M. M., \& Monteiro, C. C. F. (2015). Produção de snack extrusado com adição de farinha de bagaço de malte. Revista Tecnológica, 321-326.

Brasil. Ministério da Saúde. Agência Nacional de Vigilância Sanitária. (2002). Resolução RDC $n^{\circ} 259$, de 20 de setembro de 2002. Diário Oficial [da] República Federativa do Brasil, Brasília.

Brasil. Ministério da Saúde. Agência Nacional de Vigilância Sanitária. (2003). Resolução RDC $n^{\circ} 360$, de 23 de dezembro de 2003. Diário Oficial [da] República Federativa do Brasil, Brasília.

Brasil. Agência Nacional de Vigilância Sanitária. (2010). Dispõe sobre a oferta, propaganda, publicidade, informação e outras práticas correlatas cujo objetivo seja a divulgação e a promoção comercial de alimentos considerados com quantidades elevadas de açúcar, de gordura saturada, de gordura trans, de sódio, e de bebidas com baixo teor nutricional, nos termos desta Resolução, e dá outras providências (Resolução RDC n²4, de 15 de junho de 2010). Diário Oficial [da] República Federativa do Brasil, Brasília.

Brasil. Ministério da Saúde. Agência Nacional de Vigilância Sanitária. (2013). Informe técnico $n^{\circ} 54$ : teor de sódio dos alimentos processados. Brasília: Ministério da Saúde. Recuperado em 17 de abril, de 2019, de http://portal.anvisa.gov.br/documents/33916/388729//nforme+T\%C3\%A9cnico+n\%C2\%BA+54+de+2013/b40c6f3f-643c-4eeda2ed-81c8b607b845

Brasil. Ministério da Saúde. Agência Nacional de Vigilância Sanitária. (2015, julho 3). Resolução da Diretoria Colegiada - RDC $\mathrm{n}^{\circ} 26$, de 2 de julho de 2015. Dispõe sobre os requisitos para rotulagem obrigatória dos principais alimentos que causam alergias alimentares. Diário Oficial [da] República Federativa do Brasil, Brasília. 
Capriles, V., \& Arêas, J. A. G. (2005). Desenvolvimento de salgadinhos com teores reduzidos de gordura saturada e de ácidos graxos trans. Food Science and Technology (Campinas), 25(2), 363-369. http://dx.doi.org/10.1590/S0101-20612005000200031

Capriles, V., \& Arêas, J. A. G. (2012). Avaliação da qualidade tecnológica de snacks obtidos por extrusão de grão integral de amaranto ou de farinha de amaranto desengordurada e suas misturas com fubá de milho. Brazilian Journal of Food Technology, 15(1), 21-29.

Carvalho, C. W. P., Ascheri, J. L. R., \& Azevedo, T. L. (2005). Elaboração de extrusados expandidos (snacks) de milho e casca de soja (Comunicado técnico, No. 78, pp. 1-2). Rio de Janeiro: EMBRAPA. Recuperado em 17 de abril, de 2019, de https://ainfo.cnptia.embrapa.br/digital/bitstream/CTAA-2009-09/8945/1/ct78-2005.pdf

Codex Alimentarius Commission. (2008). Draft revised codex standard for foods for special dietary use for persons intolerant to gluten (Appendix 3). Rome: FAO. Recuperado em 17 de abril, de 2019, de http://www.fao.org/tempref/codex/Meetings/CCNFSDU/ccnfsdu28/nf28_05e.pdf

Domingues Junior, M. A. (2012). Determinação de sódio em biscoitos comercializados na região de assis (Monografia). Instituto Municipal de Ensino Superior de Assis, Assis. Recuperado em 17 de abril, de 2019, de https://cepein.femanet.com.br/BDigital/arqTccs/0911290308.pdf

Doyle, M. E., \& Glass, K. A. (2010). Sodium reduction and its effect on food safety, food quality, and human health. Comprehensive Reviews in Food Science and Food Safety, 9(1), 44-56. http://dx.doi.org/10.1111/j.1541-4337.2009.00096.x

Farias, T. A. L., Nascimento, I. R. S., Oliveira, K. L., Ximenes, G. N. C., Campos, J. M., \& Cortez, N. M. S. (2017). Rotulagem de derivados lácteos frente as novas legislações da ANVISA. Revista Brasileira de Agrotecnologia, 7(1), 106-109.

Feitosa, M. E. S. A., \& Silva, A. P. V. (2014). Teor de sódio nos salgadinhos à base de milho e de trigo comercializados na cidade de Fortaleza, Ceará. Nutrivisa - Revista de Nutrição e Vigilância em Saúde, 1(1), 1-5. Recuperado em 17 de abril, de 2019, de https://www.revistanutrivisa.com.br/artigo-original/teor-de-sodio-nos-salgadinhos-a-base-de-milho-e-de-trigocomercializados-na-cidade-de-fortaleza-ceara/

Gendel, S. M. (2012). Comparison of international food allergen labeling regulations. Regulatory Toxicology and Pharmacology, 63(2), 279-285. PMid:22565206. http://dx.doi.org/10.1016/j.yrtph.2012.04.007

Gonçalves, M. X., Reis, L. O., Moreira, V. R., \& Santos, J. R. C. (2016). Análise comparativa do teor de sódio em salgadinhos de milho por diferentes métodos. Rio de Janeiro: ABQ. Recuperado em 17 de abril, de 2019, de http://www.abq.org.br/cbq/2016/trabalhos/10/10331-22045.html

He, F. J., \& MacGregor, G. A. (2010). Reducing population salt intake worldwide: from evidence to implementation. Progress in Cardiovascular Diseases, 52(5), 363-382.

He, F. J., \& MacGregor, G. A. (2009). A comprehensive review on salt and health and current experience of worldwide salt reduction programmers. Journal of Human Hypertension, 23(6), 363-384. PMid:19110538. http://dx.doi.org/10.1038/jhh.2008.144

Hefle, S. L., Furlong, T. J., Niemann, L., Lemon-Mule, H., Sicherer, S., \& Taylor, S. L. (2007). Consumer attitudes and risks associated with packaged foods having advisory labeling regarding the presence of peanuts. The Journal of Allergy and Clinical Immunology, 120(1), 171-176. PMid:17544097. http://dx.doi.org/10.1016/j.jaci.2007.04.013

Jorhem, L., Afthan, G., Cumont, G., Dypdahl, H. P., Gadd, K., Havre, G. N., Julshamn, K., Kåverud, K., Lind, B., Loimaranta, J., Merseburg, M., Olsson, A., Piepponen, S., Sundström, B., Uppstad, B. J., Waaler, T., \& Winnerstam, L. (2000). Determination of metals in foods by atomic absorption spectrometry after dry ashing: NMKL collaborative study. Journal of AOAC International, 83(5), 1204-1211. PMid:11048861. http://dx.doi.org/10.1093/jaoac/83.5.1204

Joshi, S. M. R., Bera, M. B., \& Panesar, P. S. (2012). Extrusion cooking of maize/Spirulina mixture: factors affecting expanded product characteristics and sensory quality. Journal of Food Processing and Preservation, 38(2), 655-664. http://dx.doi.org/10.1111/jfpp.12015

Liem, D. G., Miremadi, F., \& Keast, R. S. J. (2011). Reducing sodium in foods: the effect on flavor. Nutrients, 3(6), 694-711. PMid:22254117. http://dx.doi.org/10.3390/nu3060694

Lucas, B. F., Santos, T. D., \& Costa, J. A. V. (2016). Desenvolvimento de snacks a partir de farinhas de arroz e milho orgânicas adicionados de Spirulina. In Anais do XXV Congresso Brasileiro de Ciência e Tecnologia de Alimentos (pp. 1-6). Porto Alegre: UFRGS. Recuperado em 17 de abril, de 2019, de http://www.ufrgs.br/sbctars-eventos/xxvcbcta/anais/files/175.pdf

Mendonça, S., Grossmann, M. V., \& Verhé, R. (2000). Corn bran as a fibre source in expanded snacks. LebensmittelWissenschaft + Technologie, 33(1), 2-8. http://dx.doi.org/10.1006/fstl.1999.0601

Mitchell, J. R., \& Arêas, J. A. G. (1992). Strutural in biopolymers during extrusion. In J. L. Kokini, C. T. Ho, \& M. V. Karwe (Eds.), Food extrusion science and technology. New York: Marcel Dekker.

Muraro, A., Werfel, T., Hoffmann-Sommergruber, K., Roberts, G., Beyer, K., Bindslev Jensen, C., Cardona, V., Dubois, A., duToit, G., Eigenmann, P., Fernandez Rivas, M., Halken, S., Hickstein, L., Høst, A., Knol, E., Lack, G., Marchisotto, M. J., Niggemann, B., Nwaru, B. I., Papadopoulos, N. G., Poulsen, L. K., Santos, A. F., Skypala, I., Schoepfer, A., Van Ree, R., Venter, C., Worm, M., Vlieg-Boerstra, B., Panesar, S., Silva, D., Soares-Weiser, K., Sheikh, A., Ballmer-Weber, B. K., Nilsson, C., de Jong, N. W., \& Akdis, C. A., \& EAACI Food Allergy and Anaphylaxis Guidelines Group (2014). EAACI food allergy and anaphylaxis guidelines: diagnosis and management of food allergy. Allergy, 69(8), 1008-1025. PMid:24909706. http://dx.doi.org/10.1111/all.12429

Nascimento, C., Raupp, S. M. M., Townsend, R. T., Balsan, G. A., \& Minossi, V. (2014). Conhecimento de consumidores idosos sobre rotulagem de alimentos. Revista de Epidemiologia e Controle de Infecção, 3(4), 144-147. http://dx.doi.org/10.17058/reci.v3i4.4064 
Pieretti, M. M., Chung, D., Pacenza, R., Slotkin, T., \& Sicherer, S. H. (2009). Audit of manufactured products: use of allergen advisory labels and identification of labeling ambiguities. Journal of Allergy and Clinical Immunology, 124(2), 337-341. http://dx.doi.org/10.1016/j.jaci.2009.05.032.

Poltronieri, F., Arêas, J. A. G., \& Colli, C. (2000). Extrusion and iron bioavaibility in chickpea (Cicer arietinum L.). Food Chemistry, 70(2), 175-180. http://dx.doi.org/10.1016/S0956-7135(99)00113-9

Santana, F. C. O. (2018). Rotulagem para alergênicos: uma avaliação dos rótulos de chocolates frente à nova legislação brasileira. Brazilian Journal of Food Technology, 21, e2018032. http://dx.doi.org/10.1590/1981-6723.03218

Silva, M. O. (2014). Determinação de Sódio em chips de batata pelo método de Mohr e por espectrometria de absorção atômica com chama (Monografia). Fundação Educacional do Município de Assis, Assis. Recuperado em 17 de abril, de 2019, de https://cepein.femanet.com.br/BDigital/arqTccs/1011290367.pdf

Simons, F. E., \& Sampson, H. A. (2015). Anaphylaxis: unique aspects of clinical diagnosis and management in infants (birth to age 2 years). The Journal of Allergy and Clinical Immunology, 135(5), 1125-1131. PMid:25441636.

http://dx.doi.org/10.1016/j.jaci.2014.09.014

Smith, A. C. L. (2010). Rotulagem de alimentos: avaliação da conformidade frente à legislação e propostas para a sua melhoria (Dissertação de mestrado). Faculdade de Ciências Farmacêuticas, Universidade de São Paulo, São Paulo. Recuperado em 17 de abril, de 2019, de www.teses.usp.br/teses/disponiveis/9/9131/tde-31012011-135950/pt-br.php

Soares Júnior, M. S., Santos, T. P. B., Pereira, G. F., Minafra, C. S., Caliari, M., \& Silva, F. A. (2011). Desenvolvimento de salgadinhos extrusados a partir de fragmentos de arroz e de feijão. Semina: Ciências Agrárias, 32(1), 191-200. http://dx.doi.org/10.5433/1679-0359.2011v32n1p191

Trombini, F. R. M., Leonel, M., \& Mischan, M. M. (2013). Desenvolvimento de snacks extrusados a partir de misturas de farinha de soja, fécula e farelo de mandioca. Ciência Rural, 43(1), 178-184. http://dx.doi.org/10.1590/S0103-84782012005000123

Tsugane, S., Sasazuki, S., Kobayashi, M., \& Sasaki, S. (2004). Salt and salted food intake and subsequent risk of gastric cancer among middle-aged Japanese men and women. British Journal of Cancer, 90(1), 128-134. PMid:14710219. http://dx.doi.org/10.1038/sj.bjc.6601511

Zorzanello, B. M., \& Weschenfelder, S. (2017). Pães produzidos em escala industrial e comercializados com a denominação de "integral": análise da rotulagem e identificação dos ingredientes. C\&D-Revista Eletrônica de FAINOR, 10(3), 407-423. 